\title{
Partial atrioventricular septal defect with left atrioventricular valve aneurysm mimicking valve perforation
}

\author{
Marien Lenoir ${ }^{1}$, Holy Ranaivoson ${ }^{2}$, Anne Claire Casalta ${ }^{3}$, Loïc Macé ${ }^{1}$, Philippe Aldebert ${ }^{3}$, \\ and Alexis THERON ${ }^{3}$
}

${ }^{1}$ Assistance Publique Hopitaux de Marseille

${ }^{2}$ Universite de Fianarantsoa

${ }^{3}$ Hopital de la Timone - Deparment de Cardiologie

March 6, 2022

\begin{abstract}
Left atrioventricular valve aneurysm is rare. We present a rare case of partial atrioventricular septal defect with an extremely thin left atrioventricular valve aneurysm mimicking valve perforation. The preoperative echocardiography demonstrated severe left sided atrioventricular valve regurgitation on the "cleft" and leaflet perforation. But in per-operative, we discovered a left sided atrioventricular valve aneurysm instead of valve perforation. The "cleft" edge and a aneurysm were closed.
\end{abstract}

Partial atrioventricular septal defect with left atrioventricular valve aneurysm mimicking valve perforation

Marien Lenoir ${ }^{1}$, Holy Ranaivoson ${ }^{2}$, Anne Claire Casalta ${ }^{3}$, Loïc Macé ${ }^{1}$, Philippe Aldebert ${ }^{3}$, Alexis Theron 3

1 Department of Cardiac Surgery, Hôpital Timone, AP-HM, Marseille, France

2 Department of Cardiology, CHU Tambohobe Fianarantsoa, Antananarivo, Madagascar

3 Department of Cardiology, Hôpital Timone, AP-HM, Marseille, France

Corresponding author:

Marien Lenoir

Department of Pediatric Cardiac Surgery,

264 Rue Saint-Pierre

13385 Marseille Cedex 5 France

number: 0491380000

marien.lenoir@ap-hm.fr

Total word count: 996

\section{Keywords:}

partial atrioventricular septal, mitral valve aneurysm, aneurysm, mitral valve, mitral valve cleft

Abstract 
Left atrioventricular valve aneurysm is rare. We present a rare case of partial atrioventricular septal defect with an extremely thin left atrioventricular valve aneurysm mimicking valve perforation. The preoperative echocardiography demonstrated severe left sided atrioventricular valve regurgitation on the "cleft" and leaflet perforation. But in per-operative, we discovered a left sided atrioventricular valve aneurysm instead of valve perforation. The "cleft" edge and a aneurysm were closed.

Introduction

Left atrioventricular valve aneurysm is rare (1). Left atrioventricular valve aneurysm is most common inpatient with infective endocarditis (2). Left atrioventricular valve aneurysm is usually reported as large and well-defined aneurysm by transthoracic echocardiography (2). We present a rare case of partial atrioventricular septal defect with an extremely thin left atrioventricular valve aneurysm mimicking valve perforation. A aneurysm closed by a autologous pericardial patch and "cleft" was closed.

Case report

A 48 years-old female with partial atrioventricular septal defect diagnosed in childhood but not followed. The patient had no previous history of endocarditis or inflammation. She complained dyspnea associated with chest pain in exercise. On physical examination, we find an apical systolic murmur, but she had no signs of heart failure. The rest of the examination was normal. She had cardiomegaly on the chest X ray. The trans-esophageal echocardiography (TEE) found (with 3 dimensional):

- Ostium primum defect $15 * 35 \mathrm{~mm}$

- Atrial septal defects with ostium secundum defect $8 \mathrm{~mm}$ with exclusive left to right shunt,

- Right and left atrial dilatation

- Right ventricular dilatation with right ventricular systolic pressure at $35 \mathrm{mmHg}$

- Left atrioventricular valve (Figure 1, A) with a significant leak: a predominant central component on the "cleft".

- On left atrioventricular valve, perforation of $3 \mathrm{~mm}$ (Figure 1B) in the inferior bridging leaflet.

- Left atrioventricular valve ring: $35 * 39 \mathrm{~mm}$.

- Satisfactory subvalvular apparatus with 2 papillary muscles.

Coronarography was normal.

She underwent a median full sternotomy. Cardiac arrest was induced with anterograde cardioplegia, under cardiopulmonary bypass. The left atrioventricular valve was explored through (ostium primum) and an aneurysm was discovered on the inferior bridging leaflet (Figure $2 \mathrm{C}$ ) (Figure $2 \mathrm{~A}$ ). The aneurysm was $5 \mathrm{~mm}$ $\mathrm{x} 7 \mathrm{~mm}$, and the subvalvular apparatus was normal.

Edge to edge closure of the "cleft" was performed (Figure $2 \mathrm{~B}$ ). The aneurysm had a narrow neck arising from the $3 \mathrm{~mm}$ ostium, and it was possible to gather all of the emptied aneurysm and simply cover it with the patch. An autologous pericardium patch sutured over the aneurysm with running suture (Figure 2 B). The final saline test showed no leakage. The ostium primum and ostium secondum was closed with an autologous patch with running suture. TTE demonstrated no left atrioventricular valve leakage with no aneurvrism. The patient's postoperative course was uneventful, with a normal sinus rhythm. TTE showed no left atrioventricular valve regurgitation and no valve aneurysm at discharge and 1 year.

Comment

In the literature, there only one case report who associated a partial atrioventricular septal defect with left atrioventricular valve aneurysm. Imamura and colleagues (7) reported this association, but valve aneurysm was a tissue aneurysm closing a ventricular septal defect underneath the atrioventricular valve. The present patient had no previous history of endocarditis or inflammation. She did not have a ventricular septal defect. So, the etiology of this patient is different from those previously reported. In this clinical case, the left atrioventricular valve regurgitation jet was mainly from this "cleft" that was wide open. 
Our lesion anatomically resembled a mitral valve aneurysm. Mitral valve aneurysm is a saccular and bulging structure of the mitral leaflet that expands on systole and collapses during diastole. Thus a aneurysm of the mitral valve is rare (1).

They are usually reported as a sequelae of infective endocarditis $(3,4)$. However, the underlying mechanism for its development is not known. Probably, they are the result of valvulitis with consequent formation of granulation tissue and scar tissue that succumbed to intraventricular pressure with the formation of sac-like outpouchings (5). Mitral valve aneurysm may be induced by connective tissue diseases like Marfan syndrome osteogenesis imperfect and pseudoxanthoma elasticum (6).

Left atrioventricular valve aneurysm is a difficult diagnosis to make by echocardiography and often mimic a valve perforation due to infectious endocarditis. This mistake could be explained by the thinness of the aneurysmal membrane and also a lack of resolution of echocardiography.

We recommend surgical management of left atrioventricular valve aneurysm. This is because left atrioventricular valve aneurysm might be complicated by rupture, by thromboembolism or endocarditis. When the left atrioventricular valve repair was not possible due to severely distorted leaflets or too small healthy part of the valve, we suggest left atrioventricular valve replacement (5). But, the surgical management consists at close the left atrioventricular valve aneurysm, if the ostium of the valve aneurysm is small $(<3 \mathrm{~mm})$ direct suture (8), if it is large ( $>3 \mathrm{~mm}$ ) interposition of an autologous pericardial patch.

In conclusion, left atrioventricular valve aneurysm with partial atrioventricular septal defect is an unusual case of a valve aneurysm, with a aneurysm mimicking valve regurgitation by endocarditis. The autologous pericardium patch can be used to close the large left atrioventricular valve aneurysm.

Financial Support: We did not need financial support for this study.

Conflict of Interest: Author Marien Lenoir has no conflict of interest. Author Holy Ranaivoson has no conflict of interest. Loïc Macé has no conflict of interest. Philippe Aldebert has no conflict of interest. Anne Claire Casalta has no conflict of interest. Alexis Theron declares that he has no conflict of interest.

Authors' Statement: The adult patient signed a consent for the publication of her case report.

\section{Reference}

1. Vilacosta I, San Román JA, Sarriá C, Iturralde E, Graupner C, Batlle E, et al. Clinical, anatomic, and echocardiographic characteristics of aneurysms of the mitral valve. Am J Cardiol. 1999 Jul 1;84(1):110-3, A9.

2. Reid CL, Chandraratna AN, Harrison E, Kawanishi DT, Chandrasoma P, Nimalasuriya A, et al. Mitral valve aneurysm: clinical features, echocardiographic-pathologic correlations. J Am Coll Cardiol. 1983 Sep;2(3):460-4.

3. Halkos ME, Symbas JD, Felner JM, Symbas PN. Aneurysm of the mitral valve: a rare complication of aortic valve endocarditis. Ann Thorac Surg. 2004 Oct;78(4):e65-66.

4. Saphir O, Leroy EP. True aneurysms of the mitral valve in subacute bacterial endocarditis. Am J Pathol. 1948 Jan;24(1):83-95.

5. Gajjar TP, Desai NB. True aneurysm of anterior mitral leaflet-a rare entity. J Thorac Cardiovasc Surg. 2012 Sep;144(3):e93-95.

6. Mollod M, Felner KJ, Felner JM. Mitral and Tricuspid Valve Aneurysms Evaluated by Transesophageal Echocardiography. Am J Cardiol. 1997 May 1;79(9):1269-72.

7. Imura H, Sakamoto S, Maruyama Y, Ochi M, Shimizu K. Two-patch repair for atrioventricular septal defect with mitral aneurysm. Ann Thorac Surg. 2009 Oct;88(4):1341-3. 
8. Ohira S, Doi K, Yamano T, Yaku H. Successful repair of a mitral valve aneurysm with cleft of anterior mitral leaflet in an adult. Ann Thorac Surg. 2013 Dec;96(6):2238-40.

Figure 1: A trans esophageal 3D echocardiogram revealing a "cleft" (A) and left atrioventricular valve perforation (B) mimicking perforation by endocarditis.

Figure 2: Schema of atrioventricular valve with aneurysm before repair (A) and after repair ("Cleft" closure and close aneurysm with autologous pericardium patch) (B). (C) Intraoperativephotograph of the left atrioventricular leaflets with aneurysm. The arrow indicate valve aneurysm.

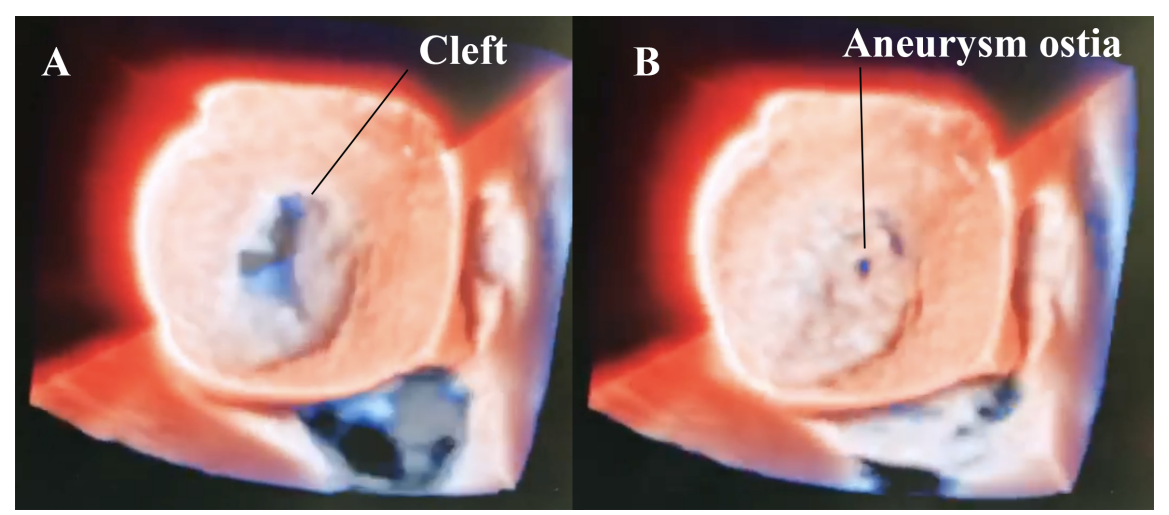



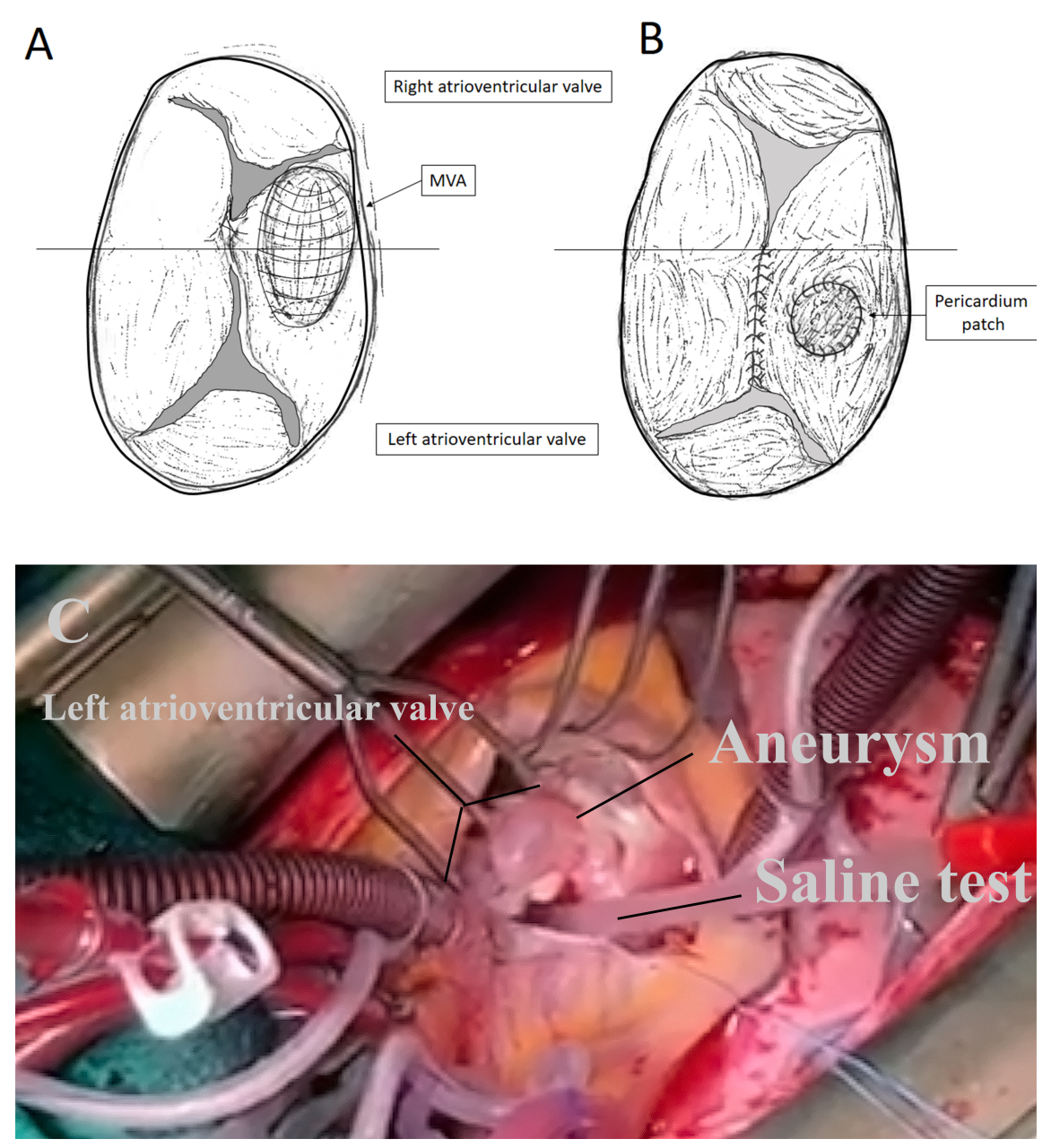